\title{
Estratigrafia sísmica de alta resolução dos sistemas deposicionais Pleistoceno Superior-Holoceno da plataforma sul fluminense, bacia de Campos.
}

*Marcela Coronel Lopes"; Antonio Tadeu dos Reis'; Clerverson Guizan Silva1 ${ }^{1}{ }^{1}$ LAGEMAR, Universidade Federal Fluminense (UFF), ${ }^{2}$ Universidade do Estado do Rio de Janeiro (UERJ).

Copyright 2014, SBGf - Sociedade Brasileira de Geofísica

Este texto foi preparado para a apresentação no VI Simpósio Brasileiro de Geofísica Porto Alegre, 14 a 16 de outubro de 2014. Seu conteúdo foi revisado pelo Comitê Técnico do VI SimBGf mas não necessariamente representa a opinião da SBGf ou de seus associados. É proibida a reprodução total ou parcial deste material para propósitos comerciais sem prévia autorização da SBGf.

\section{Abstract}

The analysis of high resolution seismic data allowed the detailed internal of shallow stratigraphic section shelf south of the Campos basin. The earthquakestratigraphic analysis revealed that the most recent sequences, last $\sim 130 \mathrm{ky}$, are architecturally more complex than revealed by previous studies. The sequence SQ4 (Latest Pleistocene) is composed by stacking two subunits seismic, Sq4A and Sq4B. These units have their top surfaces characterized by fluvial incision plumbing, indicating the occurrence of significant changes in base level during its deposition (MIS 5e and MIS 2). In sequence Sq5 were found two sedimentary patterns: the first is featured by seismic units with transparent internal facies, and presence of rare plane-parallel discontinuous reflectors, that reflects the transgressive siliciclastic sedimentation; another pattern refers to very irregular outer shape, distributed in the middle-external shelf. This facies represents carbonate buildups, developed under transgressive and highstand sea, on siliciclastic sedimentation.

\section{Resumo}

A análise de dados sísmicos de alta resolução permitiu um detalhamento interno da seção estratigráfica rasa da plataforma sul da bacia de Campos. A análise sismo-estratigráfica revelou que as sequências mais recentes, últimos $\sim 130 \mathrm{ka}$, são arquiteturalmente mais complexas que o revelado por estudos anteriores. A sequência Sq4 (Pleistoceno Superior) é composta pelo empilhamento de duas subunidades sísmicas, Sq4A e Sq4B. Essas unidades têm suas superfícies-topo caracterizadas pela incisão de canalizações fluviais, evidenciando a ocorrência de variações do nível de base durante sua deposição (MIS $5 \mathrm{e}$ e MIS 2). Na sequência Sq5 foram encontrados dois padrões sedimentares: o primeiro possui unidades sísmicas com fácies internas transparentes, e presença de raros refletores planoparalelos descontínuos, refletindo a sedimentação siliciclástica transgressiva; o outro padrão exibe forma externa bastante irregular, distribuídos na plataforma média-externa. Esta fácies representa bioconstruções carbonáticas desenvolvidas em regime transgressivo e de mar alto, por sobre a sedimentação siliciclástica.

Introdução

A bacia de Campos é uma das mais importantes bacias marginais brasileiras devido principalmente ao seu potencial exploratório. Contudo, pesquisas abordando a evolução geológica, e a estratigrafia dessa bacia durante o Quaternário permanecem relativamente escassas (ANTUNES, 1994). Estudos de estratigrafia sísmica (fonte Sparker de $700 \mathrm{~J}$ ) realizados recentemente na plataforma continental sul da bacia de Campos (Marangoni, 2012; Marangoni et. al.; 2013) permitem a visualização de detalhes da construção sedimentar interna dos depósitos quaternários correspondente a cerca de $250 \mathrm{~m}$ de espessura sedimentar (últimos $500 \mathrm{ka}$ ).

No presente estudo, a utilização de dados sísmicos de mais alta resolução permitiram o imageamento de maior resolução dos cerca de $30 \mathrm{~m}$ de espessura sedimentar superficial, o que evidenciou maiores detalhes tanto dos depósitos sedimentares, como sobre a reconstrução sedimentar da plataforma ocorridos nos últimos 130 ka (Sequência Sq4 e Sq5, Pleistoceno Superior-Holoceno).

\section{Metodologia}

Para realização deste estudo foi utilizada uma base de dados que consiste em $\sim 1.170 \mathrm{~km}$ de linhas sísmicas coletadas na área de estudo, entre Cabo de São Tomé e Cabo Frio/RJ (Fig.1). Os dados sísmicos foram adquiridos com perfilador de sub-fundo, modelo 512i da Edgetech, que opera com frequência Chirp de $0,5-5,5 \mathrm{kHz}$. Foram ainda utilizados para correlação e apoio à interpretação, dados sísmicos Sparker de $700 \mathrm{~J}$, de mais baixa resolução relativa (Fig.1).

As etapas de metodologia consistem em:

1. No pré-processamento e processamento dos dados sísmicos coletados através do programa Seismic Unix (COHEN \& STOCKWELL, 2011). Essa etapa permite a aplicação de alguns parâmetros, que são responsáveis por suprimir e atenuar ruídos (Fig.2), como: o filtro passa-banda; o MUTE; o Swell filter; o Ganho.

2. Na preparação da base de dados e na interpretação sísmica no programa PKS Kingdom Suite ${ }^{\circledR}$ (Fig.3). Após o processamento, as linhas sísmicas processadas e outros dados culturais (ex., dados geológicos, geofísicos e oceanográficos disponíveis na área) foram carregados no programa de 
interpretação sísmica PKS Kingdom Suite®. No mesmo software foi realizada a interpretação sísmica, que se baseou nos preceitos da Sismoestratigrafia e da Estratigrafia de Sequências de Alta Resolução.

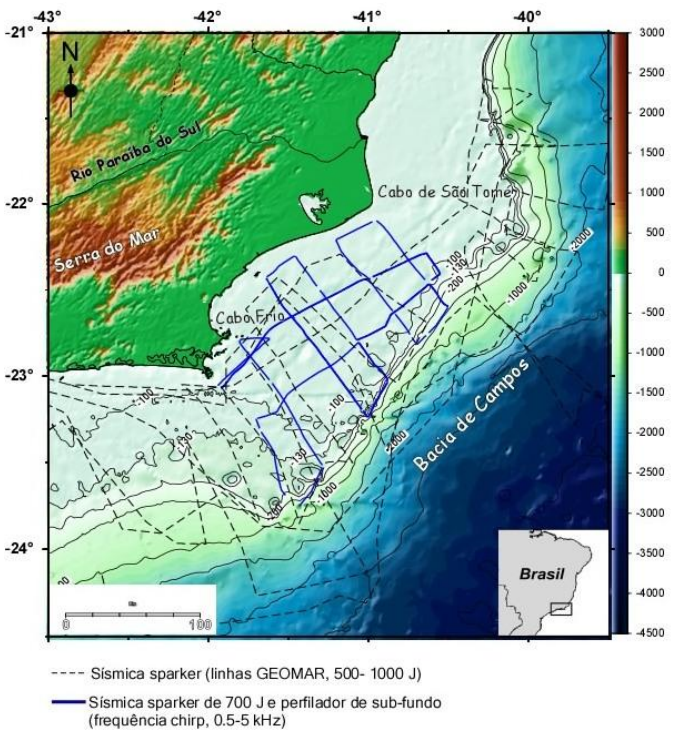

Figura 1: Mapa da plataforma continental sul da Bacia de Campos, exibindo a base de dados disponível.

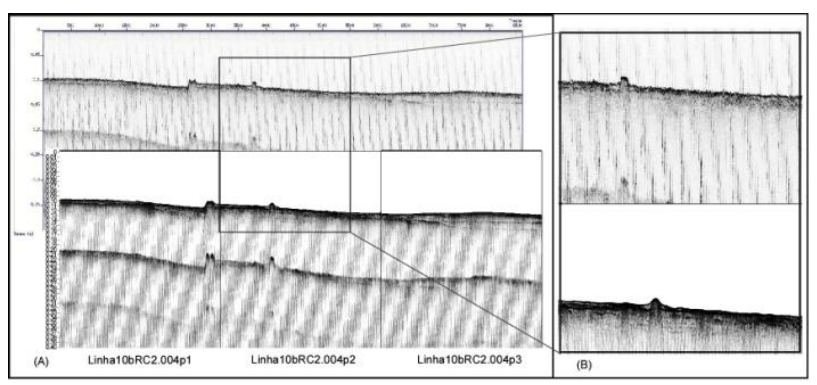

Figura 2. (A) Linha $10 \mathrm{brc2.004}$ no formato bruto (acima), e após o processamento (abaixo). (B) Ampliação de uma parte da linha com intento de melhor visualização

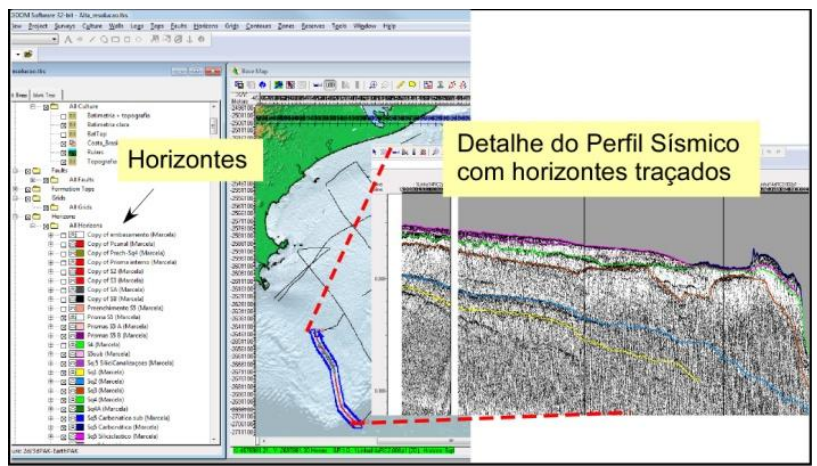

Figura 3. Demonstração da interface do software PKS Kingdom Suite ${ }^{\circledR}$, destacando os horizontes criados e suas respectivas marcações no perfil sísmico.
3. Na confecção de mapas e desenhos interpretativos. Após a interpretação sísmica foram realizados mapas evidenciando a morfologia da área de estudo, assim como foram confeccionados mapas de isópacas para a sequência estratigráfica Sq5, exibindo a variabilidade lateral de suas unidades sísmicas. Os perfis sísmicos interpretados foram exportados para o programa Canvas 11, para que cada sequência estratigráfica e subunidade fossem devidamente coloridas para a confecção de desenhos interpretativos.

\section{Resultados}

\section{Arquitetura Sísmica}

A sequência Sq4 (Pleistoceno Superior) é composta pelo empilhamento de duas subunidades sísmicas, Sq4A e Sq4B (Fig.4). Essas unidades têm suas superfícies-topo caracterizadas pela incisão de canalizações fluviais, evidenciando a ocorrência de importantes variações de nível de base entre 0 penúltimo ótimo glacial (MIS $5 \mathrm{e}$ ) e o último máximo glacial (MIS 2) (Marangoni et al., 2013).

Na unidade Sq4A, foi interpretada fácies sísmicas de preenchimento de canal (F4a), configurando o preenchimento de um canal com reflexão interna semi-transparente na base e refletores planoparalelos em direção ao topo do canal, interpretado como o registro de depósitos fluviais na base gradando no topo para influência fluvio-estuarina, refletindo um lento afogamento.

A unidade Sq4B apresenta uma espessura de $\sim 11 \mathrm{~m}$ e fácies de preenchimento de canal (F4b1) que se assemelha a F4a. Além disso, foram interpretadas as fácies F4b2 e F4b3 (Fig. 5). A fácies F4b2 exibe camada transparente, indicando deposição homogênea como a que pode ocorrer em sistemas transgressivos. Já a fácies F4b3 representa prismas sedimentares compostos por clinoformas oblíquas indicando sedimentação em contexto progradante.

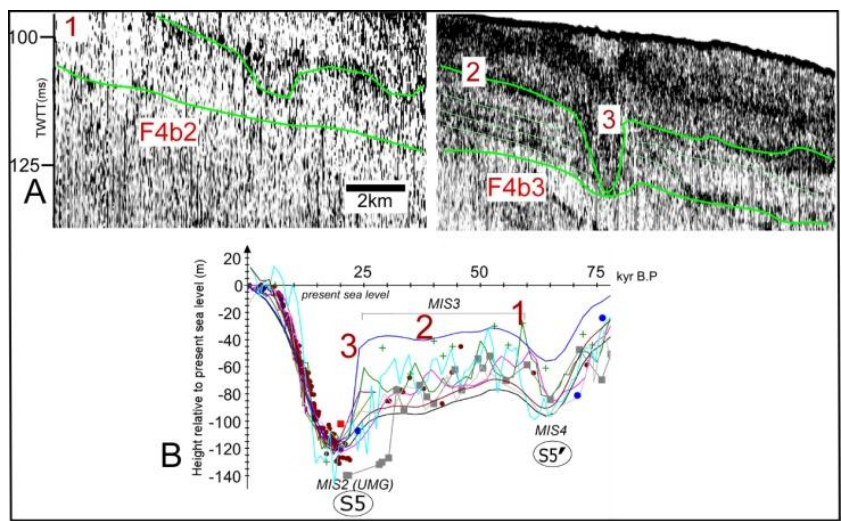

Figura 5. (A) Exemplo de perfil sísmico (perfil E Linha 16RC2), ressaltando a sequência $S q 4 b$. (B) Porção de curva global compilada por Rabineau et al. (2006), mostrando correlação do estágio isotópico MIS 3 com os eventos deposicionais no perfil sísmico em $(A)$. 
A sequência Sq5 foi muito melhor detalhada. Nessa sequência foram encontrados pelo menos 9 fácies sísmicas que refletem essencialmente a presença de dois processos sedimentares: sedimentação siliciclástica e bioconstrução carbonática (Fig.4).

A sedimentação siliciclástica transgressiva é caracterizada por: (1) fácies de preenchimento de canal (F5a1), com preenchimento plano-paralelo na base gradando para transparentes no topo, evidenciando a transição de um ambiente fluvial na base para um ambiente marinho no topo, que ocorre em condições de rápido afogamento; (2) unidades sísmicas prismáticas, com cerca de $3 \mathrm{~m}$ de espessura, e presença de refletores internos descontínuos e ondulados (F5a2) (Fig.6), que foi interpretada como a evidência de ambiente raso de alta energia refletindo a deposição de areias; (3) unidades sísmicas lenticulares, com fácies internas transparentes (F5a3 e F5a4) (Fig.7), e com presença de raros refletores plano-paralelos descontínuos (F5a5)(Fig. 6), refletindo a deposição de areias lamosas.

As bioconstruções carbonáticas, distribuídas a partir de profundidades maiores que $70-80 \mathrm{~m}$, se refere a corpos de forma externa bastante irregular: em forma de banco, com cerca de $4 \mathrm{~m}$ de espessura; e em forma pináculos, com cerca de $7 \mathrm{~m}$ espessura (Fig. 4). Essas bioconstruções podem exibir assinaturas sísmicas distintas (Fig. 4): uma fácies sísmica mais escura, com refletores internos planoparalelos, marca os carbonatos na plataforma média, indicando talvez diferentes fases de construção (F5b1); já na plataforma externa, os carbonatos exibem fácies internas transparentes sem a presença visível de refletores internos (F5b2).

2. Relação dos traços morfológicos com a sismoestratigrafia.

Os traços morfológicos encontrados foram nomeados como: Prisma progradante; Pináculos Carbonáticos; e Escarpa de 120.

o prisma progradante está posicionado na plataforma interna-média, e sua relação com a sismoestratigrafia evidencia que a região de maior rugosidade é caracterizada por fácies escuras com refletores ondulados (F5a2). Conforme o relevo se torna menos rugoso, em orientação NE-SW, tal padrão se transforma gradativamente em fácies lenticulares transparentes(F5a5) (Fig. 6.1 e 6.2).

Os pináculos carbonáticos são representados pelo lineamento presente na plataforma média-externa. Essa feição morfológica é representada pela fácies F5b1 e pode ser observada nos perfis A1, B e C (Fig. 6.1 e 6.3). A altura e largura dos pináculos são variáveis e não seguem um padrão. Esse lineamento ora se apresenta único como é mostrado pelos perfis A1 e B, e ora bifurca em duas feições lineares, o que é constatado no perfil C. A fenda que se forma possui profundidade média de $6,0 \mathrm{~m}$ e aparenta ter uma fina camada carbonática sobrepondo sedimentos siliciclásticos transgressivos finos.

A escarpa de $\sim 120 \mathrm{~m}$ representa a feição altamente erosiva na região sul, próximo à Cabo Frio, ela é delineada pela isóbata de $120 \mathrm{~m}$ (Fig. 6.1). O perfil E exibe essa feição erosiva na borda de plataforma (Fig.6.4), que foi interpretada como sendo composta por bioconstrução carbonática e por sedimentos depositados no pleistoceno (Sq4). Essa batimetria pode marcar o limite do último máximo glacial para a região de estudo. Contudo essa escarpa só é significativa na região norte, próximo ao Cabo de São Tomé e na região sul.

\section{Discussão e Conclusões}

A proposta de construção das sequências $\mathrm{Sq} 4$ e Sq5 registra tanto ciclos glacio-eustáticos de cerca de 100-120 ka, como sub-ciclos, de 20 - 40 ka do Pleistoceno Superior (Fig. 4).

Durante o estágio isotópico MIS 5 ocorreram oscilações que ajudaram a esculpir a sub-unidade Sq4A. A partir da regressão forçada no MIS 4 é iniciado o estágio isotópico MIS 3 (Fig. 4), responsável por esculpir a Sq4B. Algumas oscilações presentes nesse estágio podem ser visualizadas na figura 5. A fácies F4b2 indica deposição marinha, seguida por uma regressão, responsável por depositar sedimentos em feição progradante (F4b3). Em seguida, ocorreu uma transgressão, e após, uma regressão forçada. Evento ratificado pela presença de um canal interrompendo os refletores inclinados depositados previamente.

A Sq5 reflete a deposição de sedimentos durante a última transgressão. Para correlação com a ciclicidade deposicional foi utilizada a curva compilada por Duncan et al. (2000) (Fig. 7). A relativa lenta subida do nível do mar no início da transgressão resultou no deslocamento da linha de costa, formando antigas linhas de praia (KOWSMANN et al. 1978), e promovendo o deslocamento gradativo dos carbonatos (DELLA GIUSTINA, 2006).

$O$ caráter retrogradacional exibido na figura $7 \mathrm{~A}$ foi depositado durante uma lenta taxa de transgressão, onde as fácies sísmicas F2a, F2b, e F2c, refletem o progressivo aumento de profundidade do meio, gradando de sedimento grosso para fino (Fig. 7). Já a retrogradacão exposta na figura 7B, indica deposição durante uma mais rápida elevação do nível do mar.

O prisma progradante está associado, próximo ao Cabo de São Tomé, a mais forte hidrodinâmica, enquanto a região com morfologia mais suave, responde a uma menor hidrodinâmica local (FIGUEIREDO et al. 2011).

Os pináculos carbonáticos podem ter sido construídos em cima de escarpas esculpidas durante a desaceleração da subida do nível do mar, configurando antigas linhas de praia. $O$ padrão encontrado na fácies F5b1 pode corresponder à presença de lineamentos de barras carbonáticas sobrepondo patamares ou platôs carbonáticos (DELLA GIUSTINA, 2006).

A escarpa de $\sim 120 \mathrm{~m}$ parece marcar o limite do último máximo glacial (UMG) para a Bacia de Campos. A escarpa mais significativa à norte pode ter se formado devido a ação erosiva predominante 
da Corrente do Brasil. Já na região sul, a feição erosiva pode ser devido à uma antiga desembocadura fluvial que se formou durante o UMG (MARANGONI, 2012) (Fig.6).

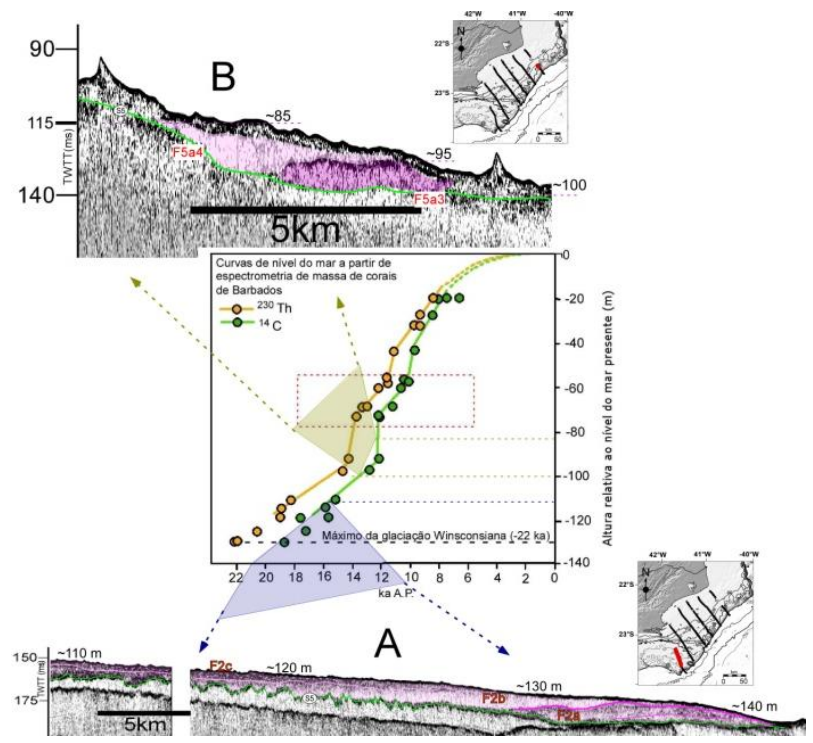

Figura 7. Feições retrogradacionais: (A) Deposição das fácies F2a, F2b, e F2c, durante uma lenta taxa de elevação do nível do mar, refletem o progressivo aumento de profundidade do meio, gradando de sedimento grosso, na base (F2a), para sedimento fino, no topo (F2c); (B) Deposição das fácies F5a3 e F5a4, refletindo a deposição de lamas transgressivas durante uma mais rápida elevação do nível do mar. As profundidades de deposição estão relacionadas com a taxa de transgressão exibida na curva compilada por Duncan et al. (2000).

\section{Agradecimentos}

Os autores agradecem ao CNPq, FAPERJ, CAPES (Ciências do Mar) e LAGEMAR.

\section{Referências}

ANTUNES, R. L., 1994. Bioestratigrafia dos nanofósseis Quaternários da Bacia de campos. B. Geoci. PETROBRAS, v. 8: 295-313.

COHEN , J.K.; STOCKWELL , J.J.W., 2001. CWP/SU: Seismic Unix: an open source software package for seismic research and processing. - Center for Wave Phenomena, Colorado School of Mines.

DELLA GIUSTINA, I. V. 2006. Sedimentação Carbonática de algas vermelhas coralináceas da plataforma continental da Bacia de Campos: Um modelo carbonático análogo para o Terciário. Dissertação (Mestrado em Geociências) Universidade Federal do Rio Grande do Sul, Rio Grande do Sul.
DUNCAN, C. S.; GOFF, J. A.; AUSTIN JR.; J. A.; FULTHORPE, C. S., 2000. Tracking the last sea-level cycle: seafloor morphology and shallow stratigraphy of the latest Quaternary New Jersey middle continental shelf. Marine Geology, v. 170: 395-421.

FIGUEIREDO JR., A. G.; PACHECO, C. E. P.; VASCONCELOS, S. C., 2011. Geomorfologia da plataforma continental da Bacia de Campos. XIII Congresso da Associação Brasileira de Estudos do Quaternário ABEQUA.

KOWSMANN, R. O.; VICALVI, M. A.; COSTA, M. P. A., 1978. Considerações sobre a sedimentação quaternária na plataforma continental entre Cabo Frio e a foz do rio Itabapoana. Rio de Janeiro, CPRM, DEGEO, REMAC. Comunicação Técnica REMAC 001/78 - relatório interno CPRM, p. 34.

MARANGONI. M. B. 2012. Arquitetura deposicional e ciclicidade dos sistemas sedimentares da plataforma continental da porção norte do estado do Rio de Janeiro - Bacia de Campos. Dissertação (Mestrado em Oceanografia) - Universidade do Estado do Rio de Janeiro, Rio de Janeiro.

MARANGONI. M. B., REIS, A. T., SILVA, C. G., MAIA, R., GUERRA, J., ARANTES-OLIVEIRA, R., PASSOS, M. BERNARDO, G. (Submetido), 2013. Arcabouço estratigráfico e ciclicidade deposicional dos sistemas sedimentares Pleistoceno Médio-Holoceno da plataforma sul da bacia de Campos, Brasil. Revista Brasileira de Geociências, 9pp.

RABINEAU, M.; BERNE S.; ASLANIAN, D.; OLIVET, J.L.; JOSEPH, P.; GUILLOCHEAU, F.; BOURILLET, J.F.; LEDREZEN, E.; GRANJEON, D., 2006. Paleo sea levels reconsidered from direct observation of paleoshoreline position during Glacial Maxima (for the last $500,000 \mathrm{yr}$ ). Earth and Planetary Science Letters, v. 252: 119-137. 


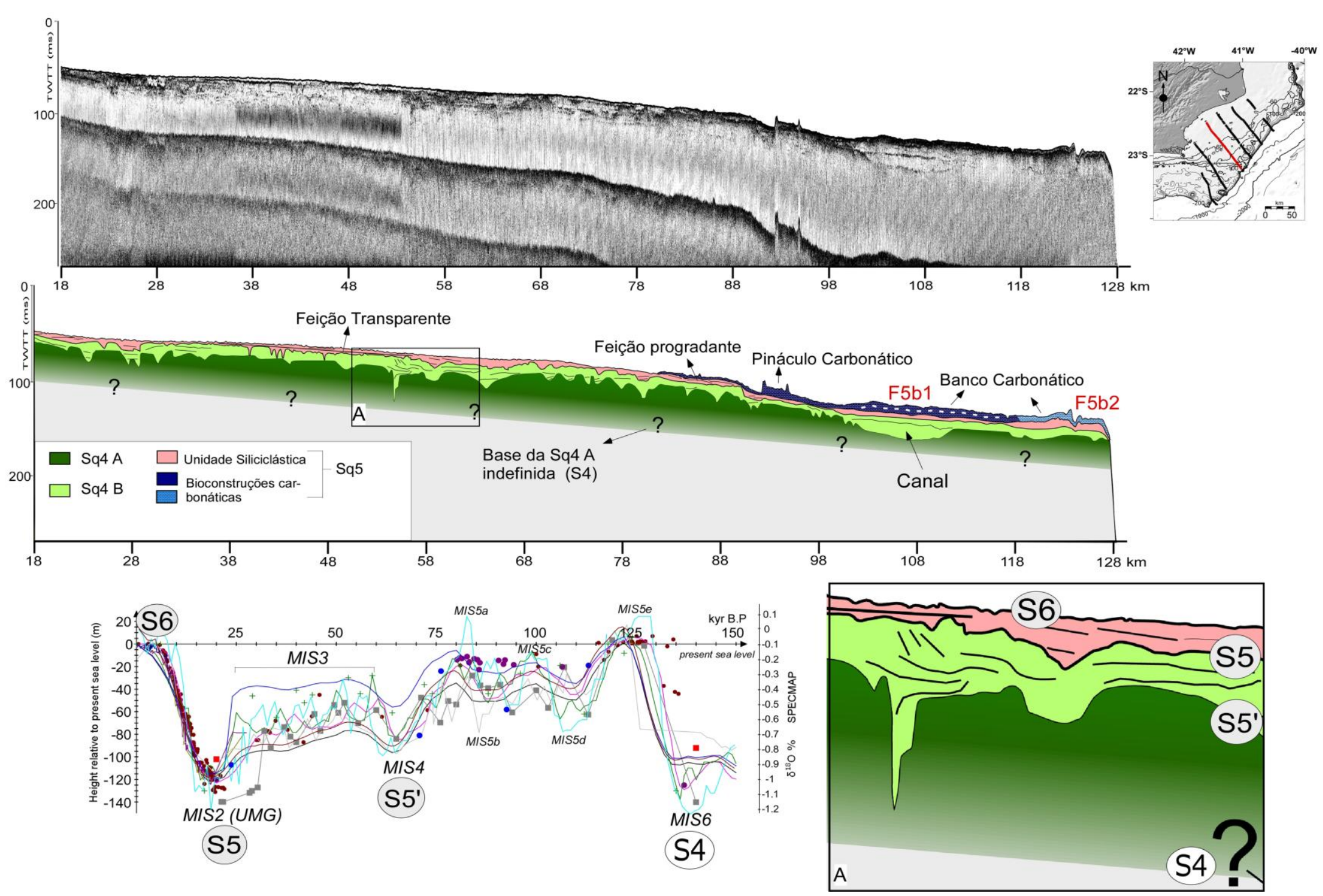

Figura 4. Exemplificação do perfil sísmico D (Linha 10RC2) ilustrando as sequências sísmicas encontradas, e suas respectivas características, como as incisões fluviais no topo da Sq4A e Sq4B, e a sedimentação siliciclástica e carbonática encontradas na Sq5. Além disso, as fácies, F5b1 - com presença de refletor plano-paralelo, e F5b2 - transparente, assinalam o padrão distinto interpretado nas bioconstruções carbonáticas. Na parte inferior é exibida a correlação entre as superfícies limites das sequências sísmicas mapeadas, com a interpretação de suas posições nas curvas isotópicas de variações eustáticas globais compiladas por Rabineau et al. (2006) 


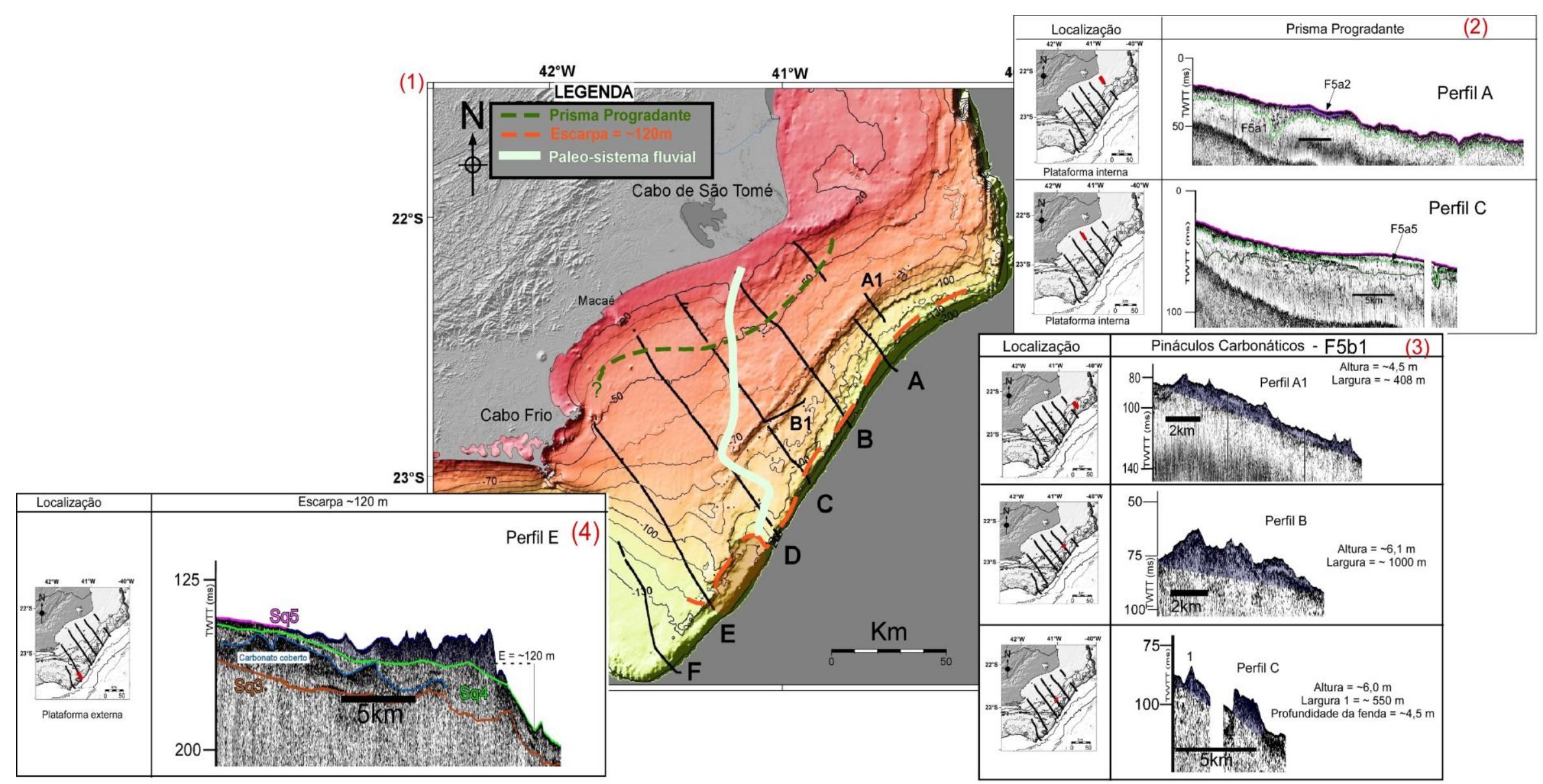

Figura 6. (1) Mapa batimétrico mostrando as características morfológicas da área de estudo e a localização dos perfis sísmicos. 0 paleo-sistema fluvial foi observado por Marangoni (2012). (2, 3, e 4) As fácies sísmicas encontradas: no prisma progradante (2); nos pináculos carbonáticos (3); na escarpa de $\sim 120 \mathrm{~m}(4)$. 